\section{Check for updates}

Cite this: Food Funct., 2019, 10, 4897

\title{
Identification and validation of common molecular targets of hydroxytyrosol $\uparrow$
}

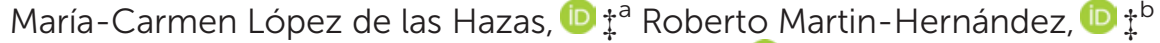 \\ María Carmen Crespo, $\$^{\mathrm{C}}$ João Tomé-Carneiro, (D) ${ }^{\mathrm{C}}$ Lorena del Pozo-Acebo, ${ }^{a}$ \\ María B. Ruiz-Roso, ${ }^{a}$ Joan C. Escola-Gil, d,e,f Jesús Osada, (D) g,h,i \\ Maria P. Portillo, (D) h,j José Alfredo Martinez, (D) h,k,l,m María A. Navarro, (D) g,h,i

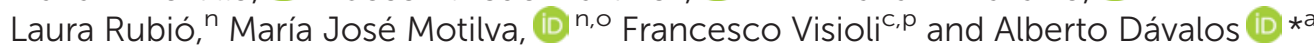

Hydroxytyrosol (HT) is involved in healthful activities and is beneficial to lipid metabolism. Many investigations focused on finding tissue-specific targets of HT through the use of different omics approaches such as transcriptomics and proteomics. However, it is not clear which (if any) of the potential molecular targets of HT reported in different studies are concurrently affected in various tissues. Following the bioinformatic analyses of publicly available data from a selection of in vivo studies involving HT-supplementation, we selected differentially expressed lipid metabolism-related genes and proteins common to more than one study, for validation in rodent liver samples from the entire selection. Four miRNAs (miR-8025p, miR-423-3p, miR-30a-5p, and miR-146b-5p) responded to HT supplementation. Of note, miR-802$5 p$ was commonly regulated in the liver and intestine. Our premise was that, in an organ crucial for lipid metabolism such as the liver, consistent modulation should be found for a specific target of HT even if

Received 31st May 2019 Accepted 13th July 2019

DOI: $10.1039 /$ c9fo01159e

rsc.li/food-function different doses and duration of HT supplementation were used in vivo. Even though our results show inconsistency regarding differentially expressed lipid metabolism-related genes and proteins across studies, we found Fgf21 and Rora as potential novel targets of HT. Omics approaches should be finetuned to better exploit the available databases.

\section{Introduction}

3,4-Dihydroxyphenylethanol (hydroxytyrosol, HT), the main olive oil phenolic compound, is mostly found as part of complex (poly)phenols (secoiridoids), ${ }^{1}$ which are easily hydrolyzed to yield HT after ingestion. ${ }^{2}$ As digestion progresses, HT- derived metabolites (mainly its sulfate form) become the main compounds circulating in blood ${ }^{3,4}$ and are recovered in urine. ${ }^{5}$ The biological properties of HT have been widely investigated in different research areas including nutrition, medicine, pharmacology, chemistry and biotechnology. ${ }^{6}$ This phenol is now considered as one of the most bioactive natural mole-

\footnotetext{
${ }^{a}$ Laboratory of Epigenetics of Lipid Metabolism, Instituto Madrileño de Estudios Avanzados (IMDEA)-Alimentación, CEI UAM+CSIC, 28049 Madrid, Spain. E-mail: alberto.davalos@imdea.org; Tel: +34912796985

${ }^{b}$ Bioinformatics and Biostatistics Unit, IMDEA Food Institute, CEI UAM+CSIC, Madrid 28049, Spain

${ }^{c}$ Laboratory of Functional Foods, Instituto Madrileño de Estudios Avanzados (IMDEA)-Alimentación, CEI UAM+CSIC, 28049 Madrid, Spain

${ }^{d}$ Institut d'Investigacions Biomèdiques (IIB) Sant Pau, Barcelona, Spain ${ }^{e}$ CIBER de Diabetes y Enfermedades Metabólicas Asociadas (CIBERDEM), Barcelona, Spain

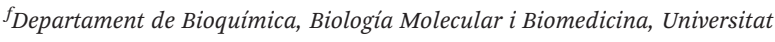
Autònoma de Barcelona, Spain

${ }^{g}$ Instituto Agroalimentario de Aragón, CITA-Universidad de Zaragoza, E-50013, Spain ${ }^{h}$ CIBER de Fisiopatología de la Obesidad y Nutrición, Instituto de Salud Carlos III, Madrid, E-28029, Spain

${ }^{i}$ Departamento de Bioquimica y Biología Molecular y Celular, Facultad de Veterinaria, Instituto de Investigación Sanitaria de Aragón-Universidad de Zaragoza, Zaragoza, E-50013, Spain
}

${ }^{j}$ Obesity and Nutrition Group. Dpt. Nutrition and food Science,

Faculty of Pharmacy, University of the Basque Country and Lucio Lascaray Research Center, Spain

${ }^{k}$ Department of Nutrition, Food Science and Physiology and Centre for Nutrition Research, University of Navarra, 31008 Pamplona, Spain

${ }^{l}$ Navarra Institute for Health Research (IdiSNA), 31008 Pamplona, Spain

${ }^{m}$ Precision Nutrition and Cardiometabolic Health Department, Madrid Institute of Advanced Studies (IMDEA Food), 28049 Madrid, Spain

${ }^{n}$ Food Technology Department, XaRTA-TPV, Escuela Técnica Superior de Ingeniería Agraria, University of Lleida, Av/Alcalde Rovira Roure 191, 25198 Lleida, Spain ${ }^{o}$ Instituto de Ciencias de la Vid y del Vino (ICVV) (Consejo Superior de Investigaciones Científicas, CSIC-Universidad de la Rioja-Gobierno de La Rioja), Logroño, Spain

${ }^{p}$ Department of Molecular Medicine, University of Padova, Viale G. Colombo 3, 35121 Padova, Italy

$\dagger$ Electronic supplementary information (ESI) available. See DOI: 10.1039/ c9fo01159e

$\$$ These authors contributed equally to this work. 
cules. ${ }^{7}$ In vitro, HT is an antioxidant ${ }^{8}$ and its intake may beneficially influence cardiovascular disease (CVD) risk, via its potential to induce anti-atherosclerotic, ${ }^{9}$ hypotensive, antioxidant, anti-inflammatory, ${ }^{10}$ and hypocholesterolemic effects. ${ }^{11}$ Of note, the European Food Safety Authority (EFSA) issued a health claim based on consistent results regarding the protective effects of olive polyphenols against the oxidation of blood lipids. ${ }^{11}$

Investigations that use cutting-edge, high-throughput, techniques referred to as "omics" are increasingly popular. ${ }^{12}$ Omics tools have allowed the deepening of the knowledge on metabolism changes and identifying new potential disease (such as CVD) biomarkers, in a way that was not possible by genetic techniques alone. ${ }^{13}$ In this sense, dietary intervention studies have successfully used transcriptomics and proteomics to show the mode through which diet induces alterations in gene and protein expression, providing information about the mechanisms of action and pathways regulated by micronutrients and helping in the identification of new biomarkers. ${ }^{14}$ However, in contrast to other fields of study, ${ }^{15}$ very few initiatives focusing on the establishment of databases that integrate largescale nutritional and genomics or genetics data have been developed. ${ }^{15-17}$ The majority of such actions are aimed at standardizing nutritional studies and some publications sometimes - over-emphasize the results obtained via database interrogation. Indeed, the scientific literature only describes two examples of such large-scale nutritional genomic data analysis: one related to functional genomics in chicken (Dhanasekaran et al., 2014) ${ }^{18}$ and the other one to genomic responses triggered by food bioactive compounds. ${ }^{12}$ Indeed, it is complicated to extract the most relevant information from the large amount of data being produced worldwide, although such an approach would greatly strengthen scientific conclusions. ${ }^{19}$

By integrating transcriptomic and proteomic data, our initial goal was to identify consistently modulated potential molecular targets of HT reported in different studies where this compound was supplemented in vivo. We then used liver samples, a key tissue in lipid metabolism, obtained from different HT rodent studies to evaluate whether the previously identified candidates could be considered as solid targets of HT.

\section{Materials and methods}

\subsection{Data collection and gene selection}

From the PubMed and Scopus scientific databases, we gathered studies on in vivo supplementation with HT or its phenolic precursors, where gene and protein differential expressions were screened. Specific queries were launched with keywords such as "hydroxytyrosol AND proteomic", "hydroxytyrosol AND transcriptomic", "hydroxytyrosol AND gene", "hydroxytyrosol AND protein", "hydroxytyrosol AND miRNA", "hydroxytyrosol AND mRNA", "hydroxytyrosol AND genomic". We generated Venn diagrams of data from the selected studies, by means of in-house $\mathrm{R}$ scripts, to find intersections among differentially expressed genes.

Functional enrichment. Genecodis3 software was used for functional enrichment using default parameters and selecting GO biological processes as target annotations.

Statistical analysis. Moderated $t$-test statistics were applied to microarray features once a linear model was fitted. Statistical significance of the overrepresented GO biological processes in the target gene list was assessed through the chisquare test. The false discovery rate (FDR) method was employed to adjust the obtained $p$-values.

\subsection{Ethics statements}

All animal studies were approved by the respective Animal Ethics Committee of the institutions where each animal experimentation took place, namely: University Complutense of Madrid (CEA-UCM 93/2012; CEEA 10-06/14, 31 ${ }^{\text {st }}$ July 2014), University of Lleida, and Universidad Mixta de Investigación, Zaragoza. All procedures followed the Guide for the Care and Use of Laboratory Animals, published by the US National Research Council (Eighth Edition, 2010), except for the study by Acin et al. (Study 3, see below), which is prior to 2010 and followed the Ethical Committee for Animal research of the University of Zaragoza.

\subsection{Brain and liver microarray analysis}

Gene expression profiles in brain and liver tissues were analyzed using the Illumina MouseRef-8 v2 Expression BeadChip ${ }^{\circledR}$ platform with Ambion Labelling. Four biological replicates per group were included. This Beadchip targets approximately 25600 well-annotated RefSeq transcripts, representing over 19100 unique genes. Data were background-corrected and normalized using GenomeStudioTM Software (Illumina, San Diego, CA, USA) and following the manufacturer's instruction. Differential expression was assessed using the Limma's Bioconductor package in the $\mathrm{R}$ statistical programming environment.

\subsection{Liver samples used for transcriptomic and proteomic validations}

Transcriptomic and proteomic validations were performed in liver samples obtained from a selection of four previously published studies where HT was administered to rodents, as follows: (1) study from Tomé-Carneiro et al. (2016), hereon referred to as Study 1. Briefly, in this study young C57BL/ 6 mice ( 2 months old, $n=14$ ) were fed a purified control diet alone $(n=7)$ or supplemented with approximately $45 \mathrm{mg}$ HT per kg bw per day (Seprox Biotech, Madrid, Spain) $(n=7)$, for 8 weeks. $^{20}$ (2) A second cohort from the Tomé-Carneiro et al. $(2016)^{20}$ study, hereon referred to as Study 2. Briefly, in this acute ingestion study, $15 \mathrm{mg}$ of HT dissolved in water (Seprox Biotech, Madrid, Spain) were administered (by gavage) to young C57BL/6 mice (10 weeks old), which were sacrificed immediately (control group, $n=9$ ) or $4 \mathrm{~h}$ after ingestion ( $n=$ 9). (3) Study from Acín et al. (2006), hereon referred to as Study 3. Briefly, for 10 weeks, 14 homozygous apoE KO mice 
( 2 months old, $n=14$ ) were given free access to drinking water (control group, $n=7$ ) or to an aqueous solution and provided a dose of $10 \mathrm{mg}$ HT per $\mathrm{kg}$ per day $(n=7) .^{21}$ (4) Study from Catalán et al. (2016), hereon referred to as Study 4. Briefly, female Wistar rats (300-350 g, $n=8$ ) were fed a standard diet (SD) (control group, $n=4$ ) or SD supplemented with $5 \mathrm{mg}$ of secoiridoids per kg per day $(n=4)$, for 21 days. $^{22}$

\subsection{Transcriptomic validations}

Commonly differentially expressed genes identified in the Venn diagram (Fig. 1) were used for transcriptomic validations. Oligonucleotide primers were designed to amplify the selected genes in both mouse and rat species. Gene function and primers are listed in Table 1. Briefly, total RNA was isolated from liver samples using a Trizol/Qiagen RNeasy kit. cDNA synthesis was performed with $1 \mu \mathrm{g}$ of RNA using the miScript II RT kit (Qiagen) according to the supplier's instructions. qPCR was carried out in a ABI 7900 HT Real-time PCR system with a 384 well plate format, using the FastStart Essential DNA Green Master mix (Roche, Switzerland) at $95{ }^{\circ} \mathrm{C}$ for $10 \mathrm{~min}$, followed by 40 cycles at $95{ }^{\circ} \mathrm{C}$ for $15 \mathrm{~s}$ and $58{ }^{\circ} \mathrm{C}$ for $1 \mathrm{~min}$. Gene expression was normalized with respect to Gapdh expression, and relative quantification was calculated using the $2^{\Delta \Delta \mathrm{Ct}}$ method.

\subsection{Proteomic validations}

For proteomic validations, protein selection was based on the commonly differentially expressed proteins identified in the corresponding Venn diagram (Fig. 3). Antibodies used and functions of the selected proteins are described in Table 2. Briefly, the liver samples from the four above-mentioned studies were homogenized in RIPA buffer (200 mM sodium orthovanadate, $1 \mathrm{mM}$ Pefabloc SC, and $2 \mathrm{mg} \mathrm{mL}^{-1}$ protease inhibitor cocktail (Sigma, Madrid, Spain)), sonicated for $5 \mathrm{~min}$ and frozen overnight at $-80^{\circ} \mathrm{C}$. After centrifugation at $12000 \mathrm{~g}$ for $30 \mathrm{~min}$ at $4{ }^{\circ} \mathrm{C}$, the supernatant was collected for total protein quantification using a Pierce BCA Protein Assay kit (Thermo Fisher Scientific) according to the manufacturer's protocol. For western blotting, $30 \mu \mathrm{g}$ of total protein were electrophoresed in 6-15\% SDS-PAGE gel and then transferred onto nitrocellulose membranes. The membranes were blocked with LiCor blocking buffer at RT for $1 \mathrm{~h}$. Overnight incubation at $4{ }^{\circ} \mathrm{C}$ was performed with a primary antibody followed by incubation ( $1 \mathrm{~h}, \mathrm{RT}$ ) with the LiCor fluorescent secondary antibody (goat anti-Mouse IRDye® $800 \mathrm{CW}$ or goat anti-Rabbit IRDye® $680 \mathrm{CW}$ ). The membranes were visualized using a LiCor Odyssey scanner. Proteins were quantified by densitometry using Image Studio Lite 5.2.5 analytical software (LiCor, Lincoln, NE) and normalized to GAPDH.

\section{7 miRNA analysis}

For miRNA analysis, an unbiased whole genome miRNA analysis was performed in the mice liver samples ( $n=5$ per group) from Study 1 using small RNA sequencing. RNA integrity was determined using an Agilent 2100 Bioanalyzer (Agilent Technologies, Santa Clara, CA). Following the manufacturer's protocol, a NEBNext巴 multiplex small RNA Library Prep Set for Illumina (New England BioLabs, Ipswich, MA) was used to prepare the libraries, and sequenced using the Illumina NextSeq 500 platform. After trimming adapter sequences, Bowtie2 was used for read alignment against high confidence mouse mature miRNA sequences, obtained from the miRBase

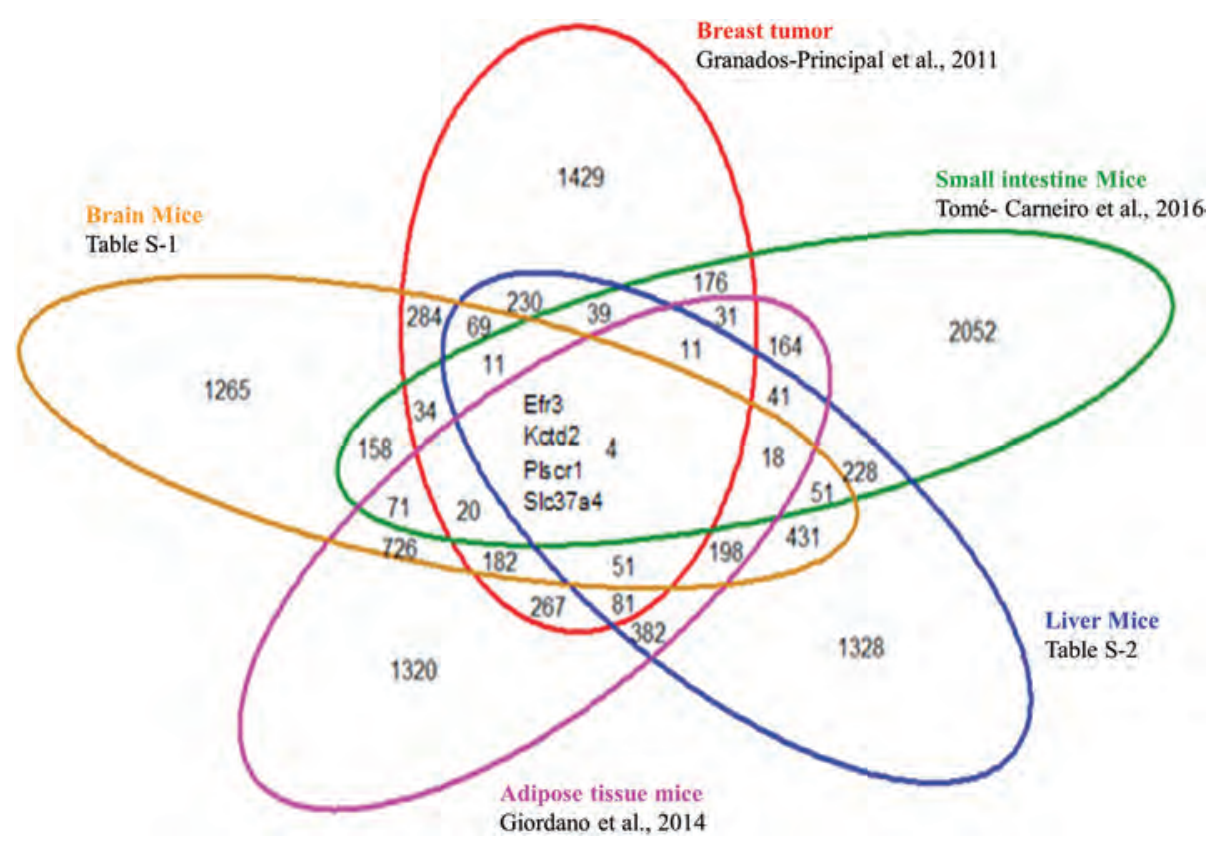

Fig. 1 Venn diagram showing the common differentially expressed genes identified through bioinformatic analysis. Transcriptomic data available in public repositories from in vivo dietary supplementation with hydroxytyrosol. Gene differential expression was analyzed using LIMMA models. 
Table 1 Function of the genes and list of primers designed for the validation of the studies

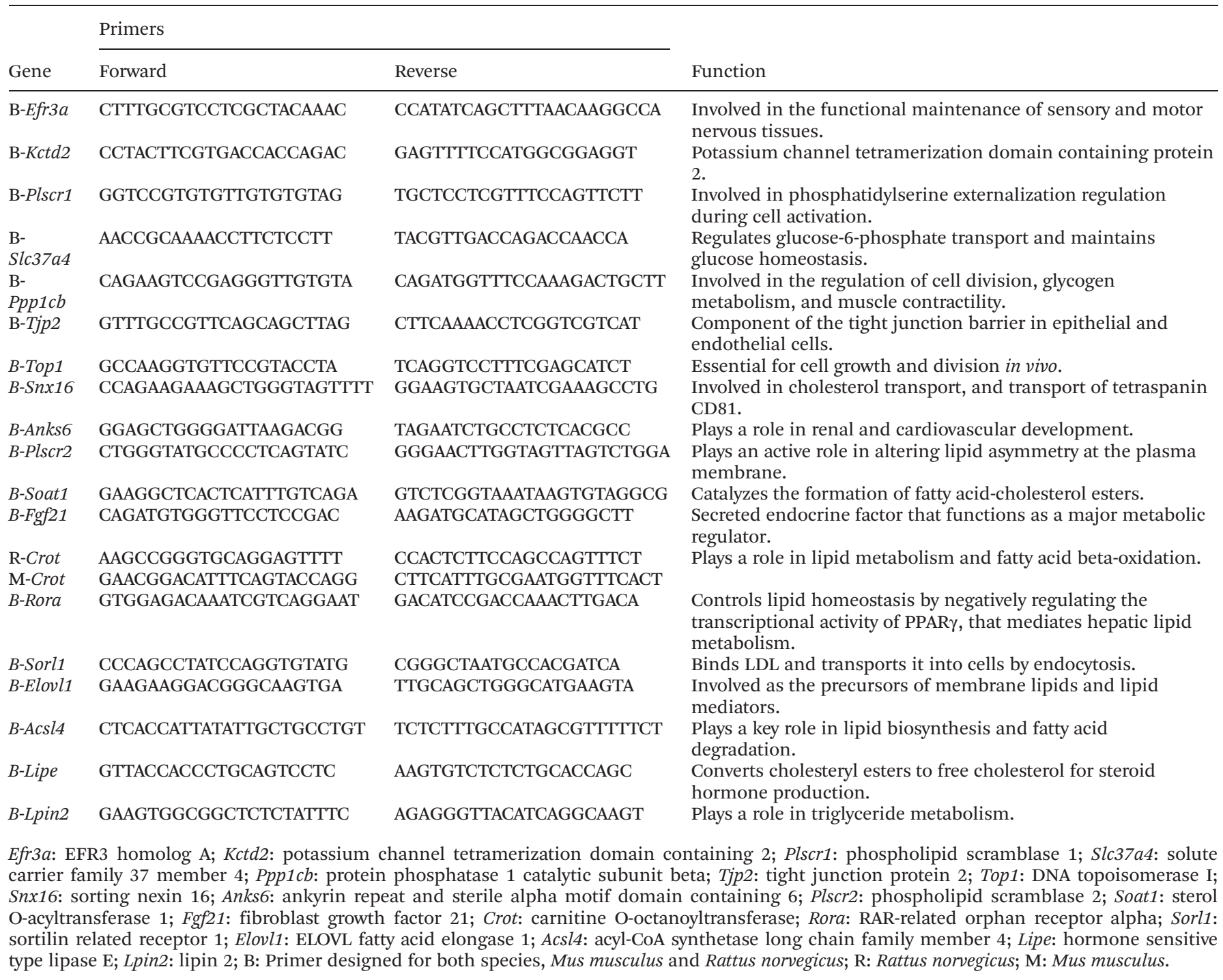

database. Finally, only reads showing a unique valid alignment against the reference sequences were considered for mature miRNA counting.

\section{8. miRNA bioinformatic analysis}

MicroRNAs' targets presenting hits on the 3'UTR position and showing a binding $P$-value score equal to 1 were obtained from the miRWalk 3.0 database ${ }^{23}$ and used for further analysis. A functional enrichment of these genes, targeted by at least two of the differentially expressed microRNAs, was performed in the Panther database v.11 ${ }^{24}$ using Gene Ontology (GO) and Panther pathway annotations. A subset of four significantly modulated miRNAs in response to HT supplementation were used for Gene Interaction (GI) analysis using the above-mentioned target genes. GI analysis was performed as previously described $^{20}$ including target genes targeted at least by two miRNAs. The target dot size was directly correlated with the number of interactions with the set of miRNAs. As for the functional analysis, only the genes targeted simultaneously by at least two miRNAs are shown.

\subsection{Statistical analysis}

Comparisons between HT-supplemented groups and controls were performed by means of two-tailed $t$ tests or MannWhitney tests; when assumptions for parametric testing were not met GraphPad Prism 7.02 (La Jolla, CA) was used. In all cases, $p<0.05$ was considered as statistically significant.

\section{Results}

\subsection{Identification of common differentially expressed genes}

Specific searches in public scientific databases for in vivo interventions involving hydroxytyrosol supplementation returned scarce results (Table 3). The GEO database, which contains high throughput genomic and proteomic data among others, 
Table 2 Selected proteins and types of antibodies used

\begin{tabular}{|c|c|c|c|c|}
\hline Proteins & Company & $\begin{array}{l}\text { Molecular } \\
\text { weight }(\mathrm{kDa})\end{array}$ & Host & Function \\
\hline CAR3 & Thermo Fisher & 29.6 & Rabbit & Involved in oxidative stress. \\
\hline PRDX1 & Cell Signaling & 21 & Rabbit & $\begin{array}{l}\text { Belongs to a family of antioxidant enzymes. Reduction of hydrogen } \\
\text { peroxide and alkyl hydroperoxides. }\end{array}$ \\
\hline VIM & Cell Signaling & 57 & Rabbit & Involved in neurogenesis and cholesterol transport. \\
\hline ACTN4 & Bethyl & 110 & Rabbit & $\begin{array}{l}\text { Transcriptional coactivator, stimulating transcription mediated } \\
\text { by the nuclear hormone receptors PPARG and RARA. }\end{array}$ \\
\hline
\end{tabular}

CAR3: Carbonic anhydrase 3; FASN: fatty acid synthase; PRDX1: peroxiredoxin 1; VIM: vimentin; HSPD1: heat shock protein family D (Hsp60) member 1; ACTN4: actinin alpha 4; GAPDH: glyceraldehyde-3-phosphate dehydrogenase.

Table 3 In vivo studies involving supplementation with hydroxytyrosol where transcriptomic analyses were performed

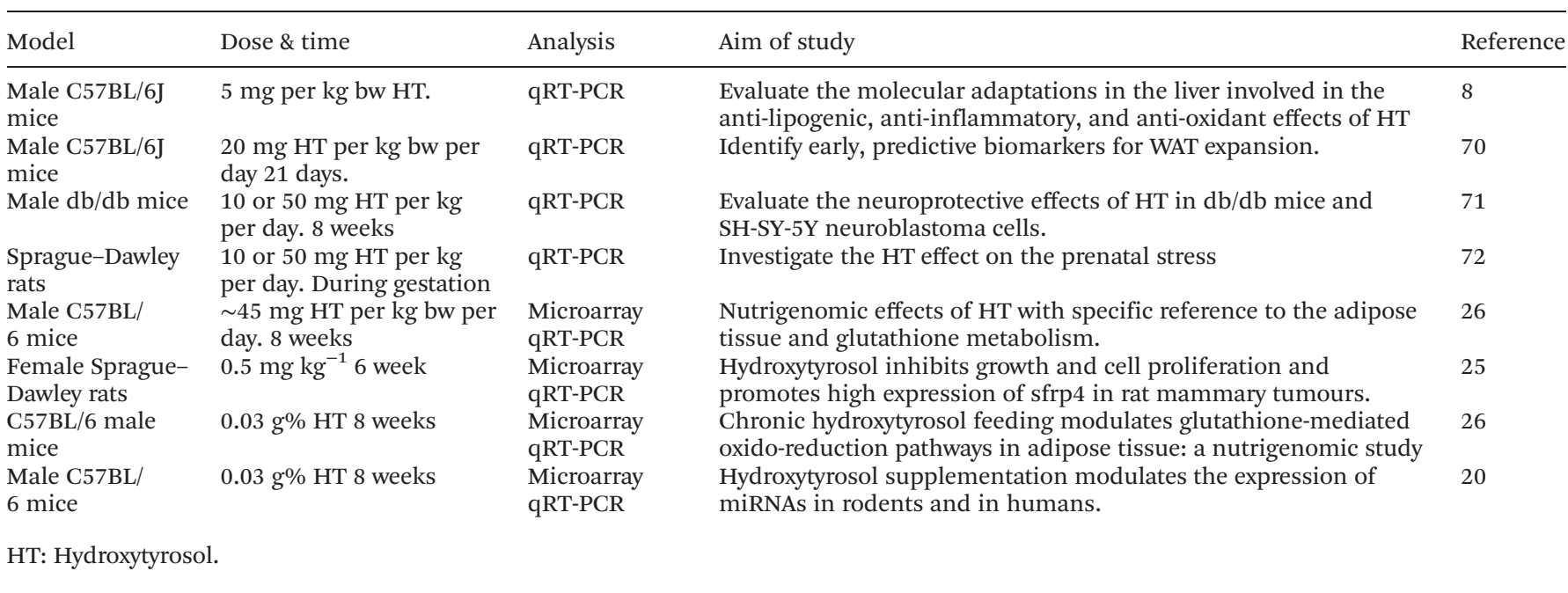

only accounted for two studies concerning gene expression in humans (GSE75027 and GSE75026) after olive oil intake (where HT is preeminent). Moreover, only one study concerning an in vivo intervention with HT was found, where a breast tumor-induced model of Rattus norvegicus was used (GSE15944). ${ }^{25}$ Finally, two sets of data from a study carried out in our laboratory (ESI Tables S1 and S2; $\uparrow$ not available in scientific databases), regarding microarray screening in brain and liver tissues from diet-HT supplemented mice, ${ }^{26}$ were included in this study. The aforementioned five sets of data were used to find mutual differentially expressed genes (Fig. 1). Efr3, Kctd2, Plscr1 and Scl37a4 were identified as differentially expressed after HT supplementation in all analyzed data sets.

\subsection{Transcriptomic validations}

After the identification of differentially expressed genes shared by at least three studies (Fig. 1 and ESI Table S $3 \dagger$ ), 18 genes associated with lipid metabolism were finally selected for validation. Validation was performed in the liver samples from HTsupplemented animal models vs. controls (Fig. 2) taken from the four selected studies (Studies 1-4, see Materials and methods for details). In the liver samples from Study 1 a statistically significant increase was seen in Tjp2, Top1, Rora, Sorl1, Fgf21 and Ppp1cb (Fig. 2A). In the liver samples from Study 2 a statistically significant increase was seen in Slc37a4, Snx16, Rora and Fgf21, whereas a statistically significant decrease was observed in Sorl1 (Fig. 2B). In the liver samples from Study 3 a statistically significant increase was seen in Slc37a4, Anks6, Plscr2 and Lipe (Fig. 2C). In the liver samples from Study 4 a statistically significant increase was seen in Sorl1 (Fig. 2D).

\subsection{Identification of common differentially expressed proteins}

Publicly available large-scale proteomics data regarding HT supplementation are scarce. To determine whether HT consumption affects specific signaling pathways, we comprehensively analyzed publications involving in vivo HT supplementation to extract protein expression information from the reported data tables (Table 4 ). Then, the collected proteomic data were subjected bioinformatic analyses to identify differentially expressed proteins common to at least two of these studies (Fig. 3). Bioinformatic analysis showed that ALDH2, SELENBP1, HSPD1, PPIA, VIM, YWHAG, RPL8, ACTN4, NPM1, 
A

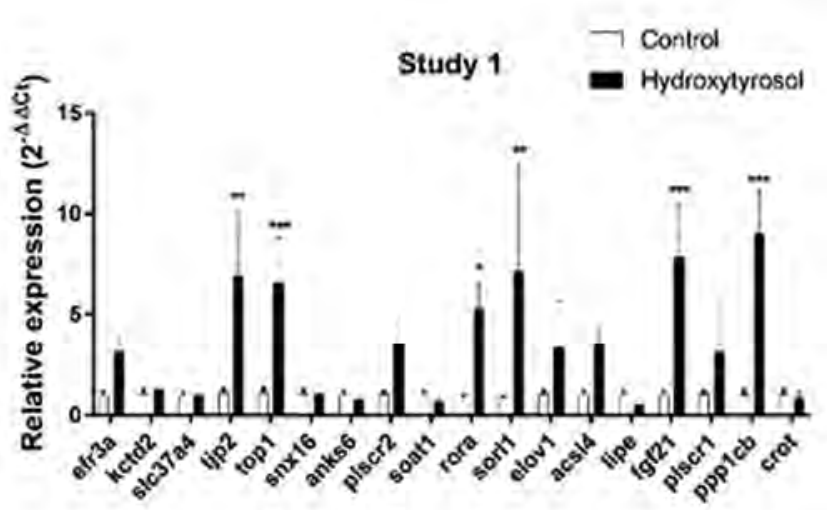

B

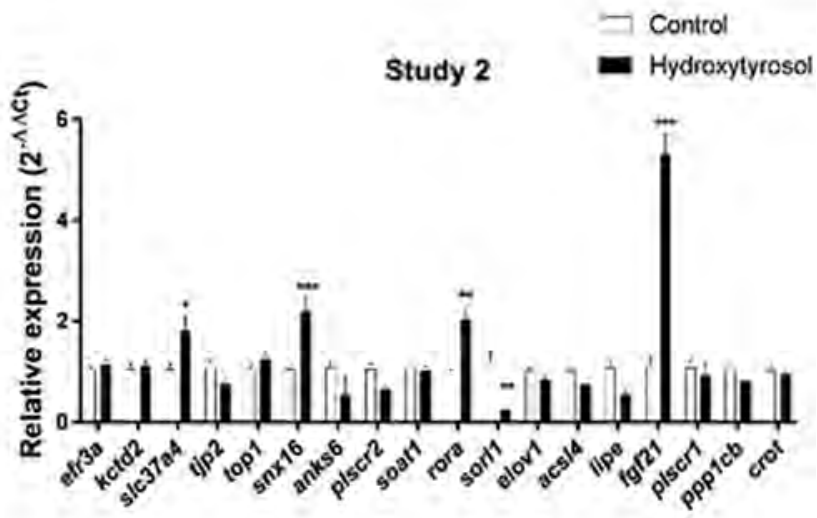

C

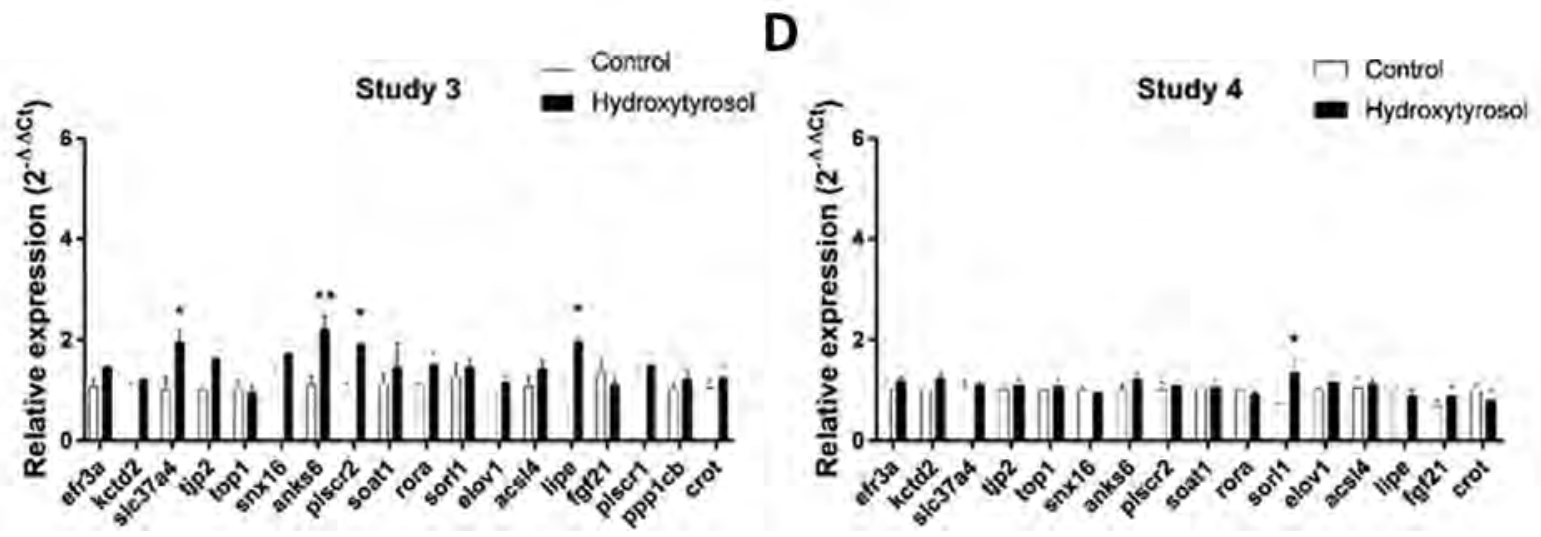

Fig. 2 Validation of common transcripts predicted to be modulated by hydroxytyrosol supplementation. A set of transcripts were chosen from bioinformatic analysis and validated in the liver samples of different intervention studies. Gene expression was analyzed by RT-qPCR. (A) Male young C57BL/6 mice ( $n=7$ per group) fed with a control or HT diet (45 mg HT per kg bw per day), for 8 weeks (Study 1). (B) Male young C57BL/6 mice were administered (gavage) an acute dose of $15 \mathrm{mg}$ of HT (dissolved in water) and sacrificed $4 \mathrm{~h}$ after ingestion ( $n=9$ ) (Study 2). (C) Male young homozygous apoE KO mice ( $n=7$ per group) fed with an aqueous solution of $10 \mathrm{mg}$ HT per kg per day ( $n=7$ ), for 10 weeks (Study 3). (D) Female Wistar rats (300-350 g, $n=4$ per group) fed with a standard diet (SD) or SD supplemented with 5 mg HT per kg per day, for 21 days (Study 4). HT, hydroxytyrosol.

Table 4 In vivo studies involving supplementation with hydroxytyrosol where high throughput proteomic analyses were performed

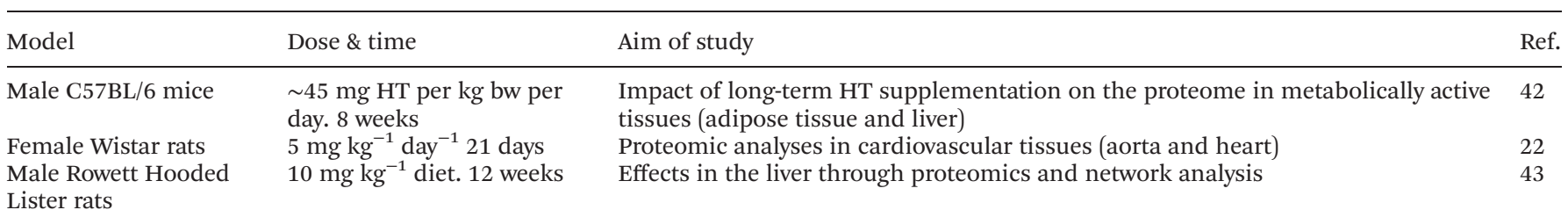

Lister rats

HT: Hydroxytyrosol; ACTN4: alpha-actinin-4; RPL8: 60S ribosomal protein L8; ALDH2: mitochondrial aldehyde dehydrogenase.

ALB, HSP90AB1, CAR3, FASN, HBB-B1, PRDX1, CFL1, GLUD1, VCL, DLAT, and GPD1 proteins were common to at least 2 studies. Only two proteins, Hspd1 and Actn4, were common to three studies.

\subsection{Proteomic validations}

After the identification of differentially expressed proteins, common to at least two studies, validation was carried out in 


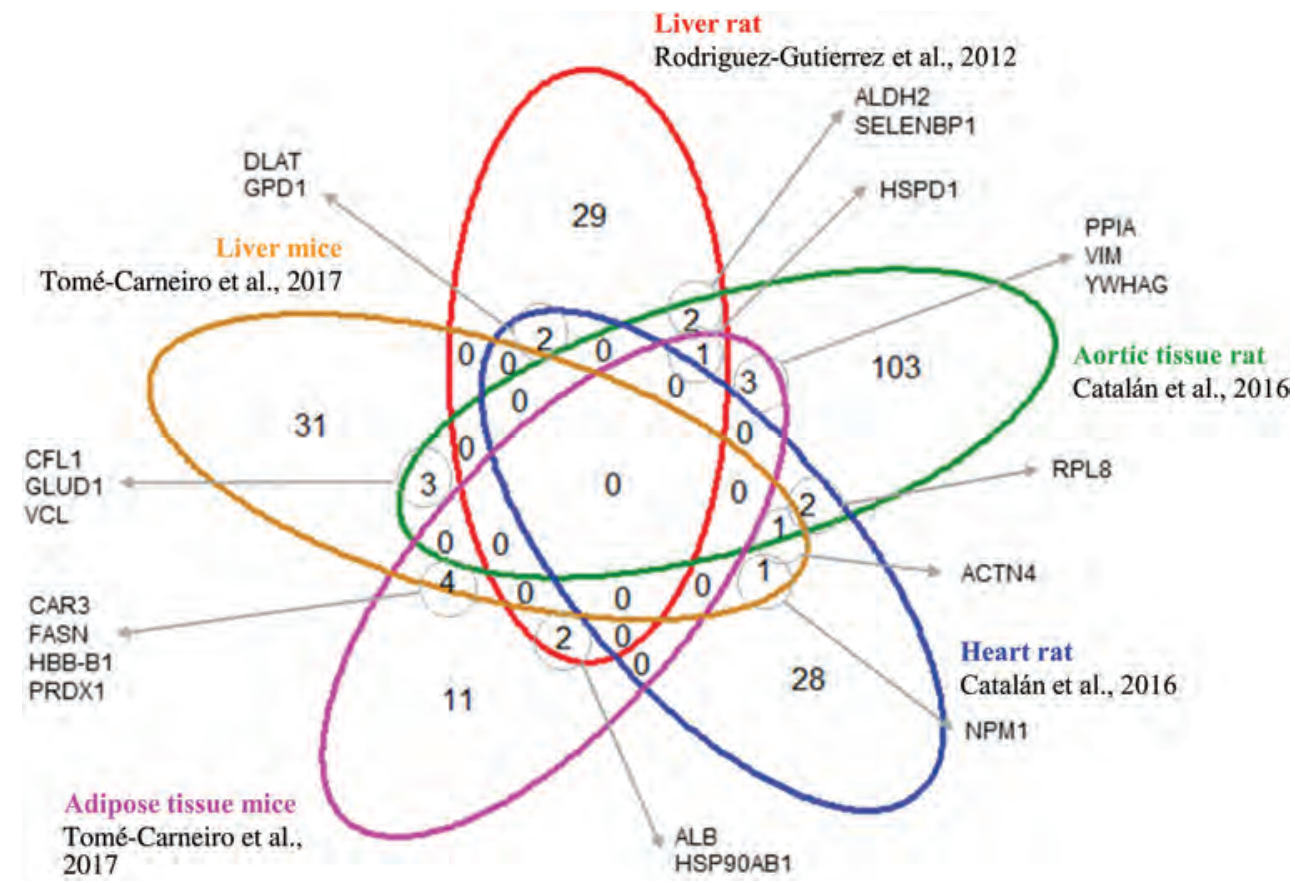

Fig. 3 Venn diagram showing the common differentially expressed proteins identified by means of bioinformatic analysis. Proteomic data available in the scientific literature from in vivo dietary supplementation with hydroxytyrosol.

the liver samples from the HT-supplemented animal models and controls (Studies 1-4). Overall, we did not find significant differences in any of the proteins analyzed in the livers of the HT- (or their secoiridoids precursors) supplemented animals compared with those of the controls (Fig. 4). In the liver samples from Study 1, the HT-supplemented groups showed a slight, statistically non-significant, decrease in VIM and increase in HSPD1, compared with those of the controls (Fig. 4A). In samples from Study 2, involving an acute ingestion of HT, a decrease in the expression was seen for PRDX1 and CAR3, and an increase in FASN, although statistical significance was not reached (Fig. 4B). As for Study 3, in HT-supplemented ApoE KO mice, non-significant decreases in the expression of ACTN4 and an increase in VIM were observed (Fig. 4C). Finally, a non-significant decrease in FASN was observed in the liver of female Wistar rats (Study 4) (Fig. 4D). CAR3 was not analyzed in Study 4 samples as the anti-mouse antibody used had no cross-reactivity to rats.

\subsection{Post-transcriptional regulation by miRNAs}

Post-transcriptional regulation is commonplace in biological systems and miRNAs bind complementarily to the $3^{\prime} \mathrm{UTR}$ sequence mediating negative post-transcriptional regulation, ${ }^{27}$ in turn impacting the proteome. ${ }^{28}$ Thus, we next assessed the modulation of miRNA levels and explored the potential impact it could have on the proteome. The liver samples from Study 1 were subjected to small RNA sequencing and miRNAs were analyzed (Fig. 5). From the 247 miRNAs detected in the mouse liver samples (ESI Table S4 $\dagger$ ), only 4 were found to be differentially expressed in the HT supplemented group after FDR adjustment (Fig. 5A). From these, miR-802-5p, miR-30a-5p and miR-146b-5p were up-regulated, whereas miR-423-3p was down-regulated. Because one gene can be regulated by different miRNAs, we also searched for validated targets likely to be modulated by more than one miRNA responding to HT treatment (Fig. 5B). Gene Interaction (GI) analysis was performed (see Materials and methods for details) generating a unique list of 279 genes potentially modulated by at least two miRNAs. The genes modulated by the four miRNAs included Ccdc117, Ntrk2, Mrpl17, Timm22, Zfp945, Ubxn7, Tmem71, Slc30a7, Gucy1a2, 4931406C07Rik, Zdhhc21, and Dclk1 (Fig. 5B). In particular, Gucy 1 a2 is involved in an endothelin signaling pathway, Zdhhc21 in metabolic processes (palmitoyltransferase), and Timm22/Ntrk2 in cellular component organization. Gene ontology analysis of modulated miRNAs targets (by more than one miRNA) suggested their involvement in the regulation of major pathways, including the Wnt signaling pathway (P00057), the CCKR signaling map (P06959) or the inflammation mediated by the chemokine and cytokine signaling pathway (P00031), among others (Fig. 5C). Finally, none of the genes matched the ones obtained after the analysis of the transcriptomic or proteomic data sets, suggesting that their levels are not directly controlled by these specific miRNAs.

\section{Discussion}

Many nutritional intervention studies ${ }^{29,30}$ demonstrated that food and its bioactive components affect the expression of genes, which can impact disease prevention. ${ }^{31-34}$ High 
A

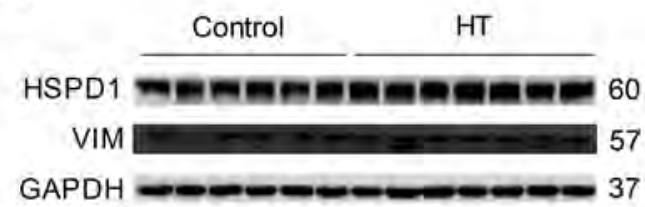

B

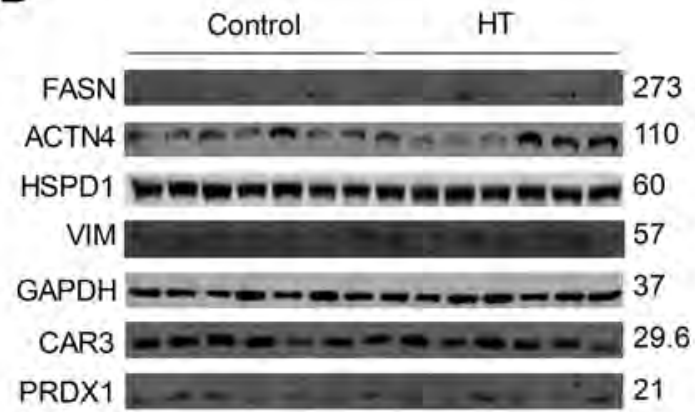

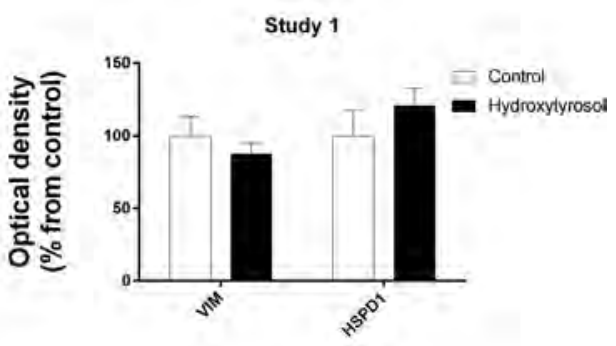

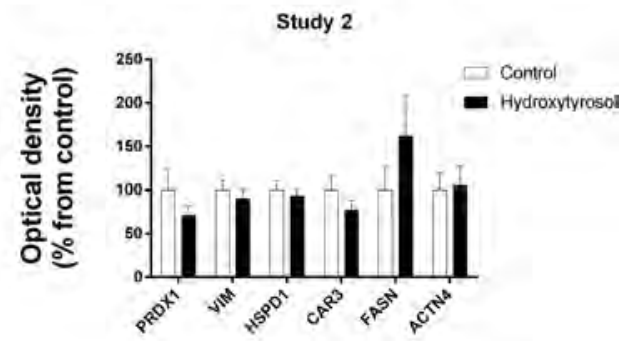

C
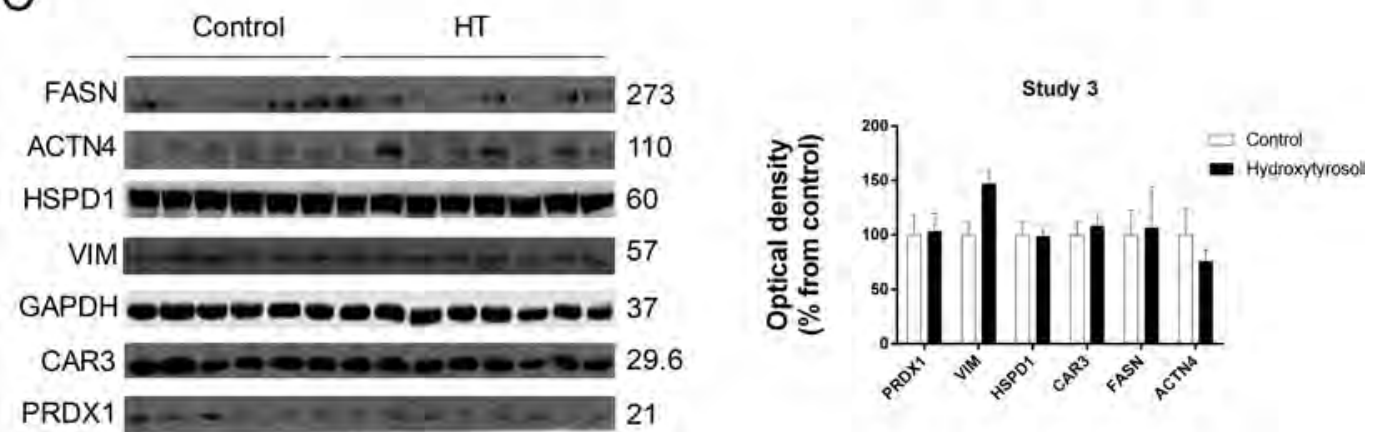

D
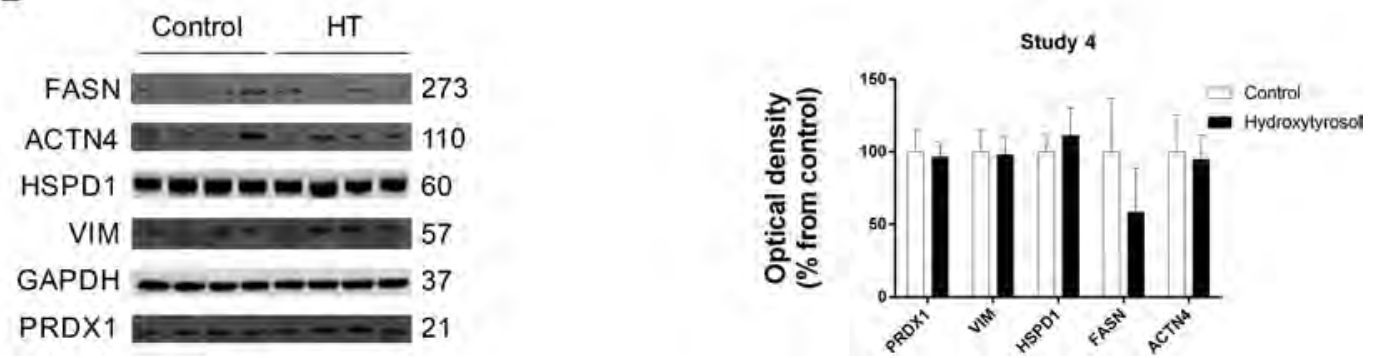

Fig. 4 Validation of common proteins predicted to be modulated by HT supplementation. A set of proteins were chosen after bioinformatic analysis of proteomic data and validated in the liver samples of different intervention studies. Protein expression was analyzed by western blotting. (A) Male young C57BL/6 mice ( $n=7$ per group) fed with a control or HT diet (45 mg HT per kg bw per day), for 8 weeks (Study 1). (B) Male young C57BL/6 mice administered (gavage) with an acute dose of $15 \mathrm{mg}$ of HT dissolved in water and sacrificed $4 \mathrm{~h}$ after ingestion ( $n=9$ ) (Study 2). (C) Male young homozygous apoE KO mice ( $n=7$ per group) fed with an aqueous solution of $10 \mathrm{mg} \mathrm{HT}$ per kg per day ( $n=7$ ), for 10 weeks (Study 3). (D) Female Wistar rats (300-350 g, $n=4$ per group) fed with a standard diet (SD) or SD supplemented with 5 mg HT per kg per day, for 21 days (Study 4). HT, hydroxytyrosol.

throughput transcriptome and proteome analysis can be very useful in the discovery of new biomarkers and pathways implicated in metabolic diseases. ${ }^{35}$ Moreover, high throughput analyses aid in assessing the physiological effect that bioactive compounds exert on a wide variety of diseases such as diabetes, ${ }^{36,37}$ obesity, ${ }^{38}$ and cancer. ${ }^{39,40}$ Hence, these tech- niques are useful to explore the mechanisms of action of nutrients and phytochemicals. Omics technologies are widely adopted to concomitantly study the expression of thousands of genes and proteins, generating a vast amount of data that accumulates over time and is generally available in public repositories. These data sets could potentially be exploited to 


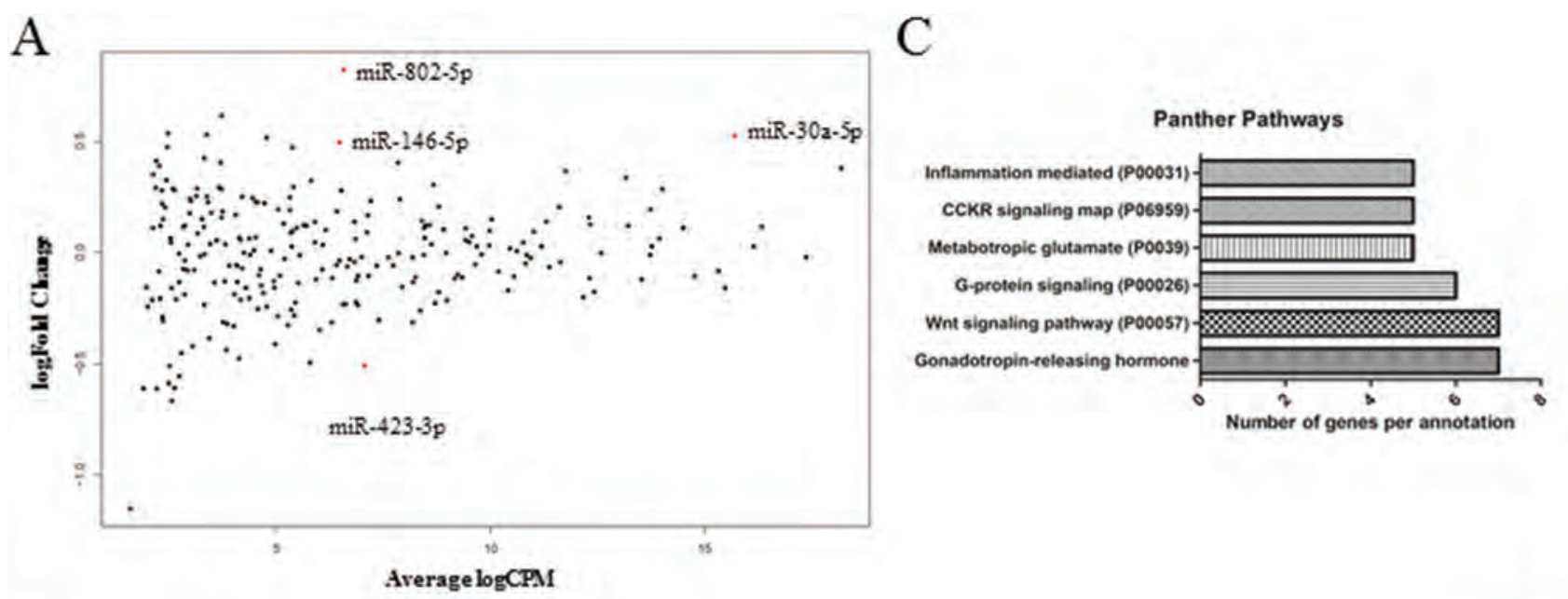

\section{B}

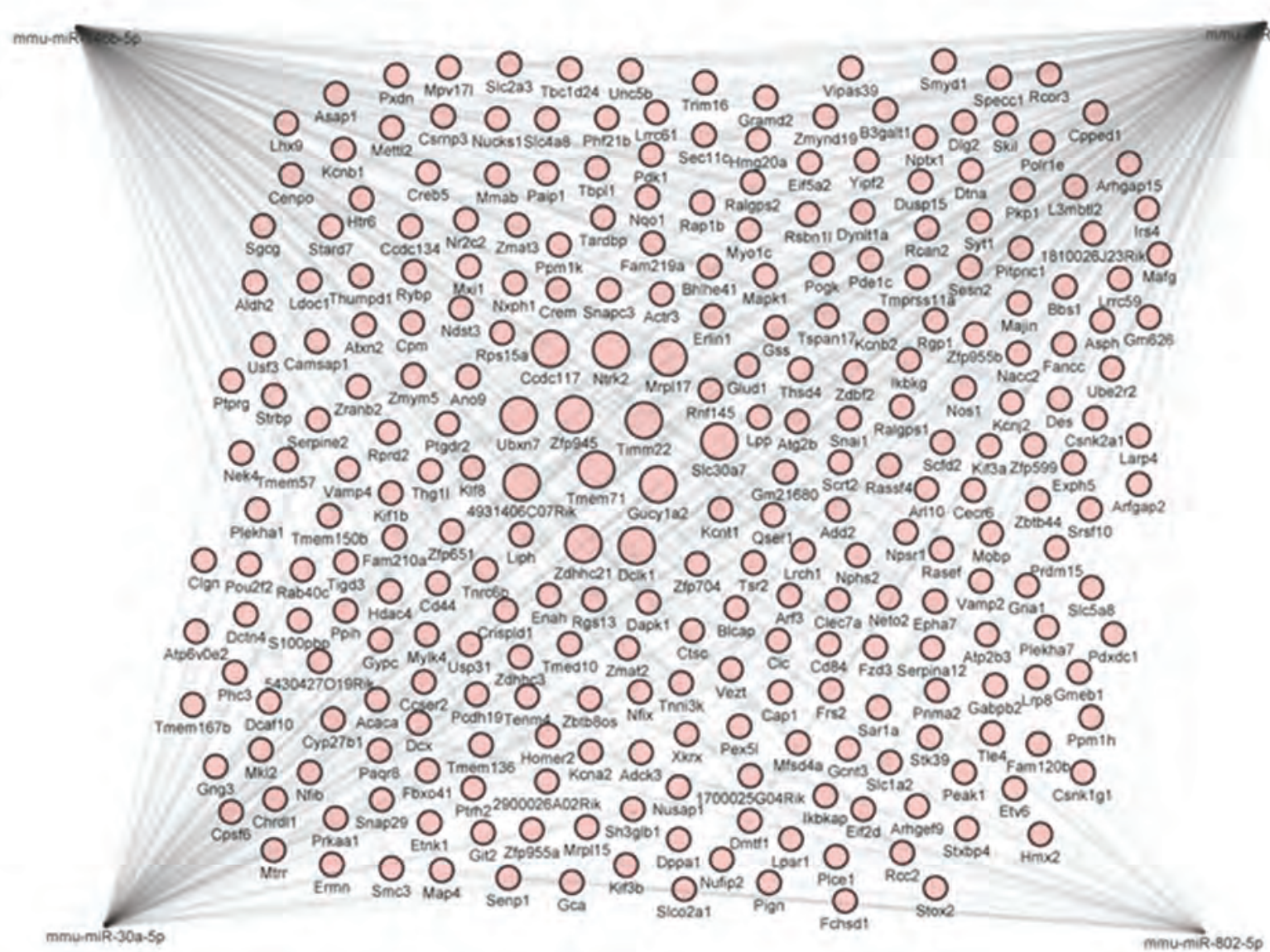

Fig. 5 Liver miRNA analysis. (A) Scatter plot of the RNA-seq data of liver miRNAs from mice supplemented with HT, for 8 weeks. (B) Genetic interaction analysis between miRNAs and their likely miRNA targets. Target point sizes are directly correlated with the number of interactions within the set of miRNAs. (C) Functional enrichment analysis of differentially expressed miRNA targets. HT, hydroxytyrosol.

establish functional connections among compounds triggering similar responses at the molecular level by using computational approaches involving machine learning tools such as hierarchical clustering. ${ }^{41}$ For example, we can predict the pharmacological properties of many molecules across different biological systems and conditions solely based on their tran- scriptional profiles. ${ }^{41}$ Yet, there are few applications of such approaches in the emerging field of nutrigenomics, which investigates the effects of food and nutrients on gene expression. ${ }^{12}$ Here, we analyzed the available transcriptomic and proteomic data to (1) identify differentially expressed genes and proteins prevailing among studies addressing 
hydroxytyrosol supplementation in vivo and (2) validate the identified differentially expressed genes and proteins as robust targets of HT.

We first searched for in vivo experiments involving dietary supplementation with HT where high throughput gene and protein expression data were generated. Then, we identified a signature of dozens of genes shared among the selected studies which could be related to the biological effects associated with HT consumption. Although several genes exhibited different expressions in at least two tissues, only four transcripts were significantly modulated in the four tissues we analyzed, i.e. brain, adipose tissue, liver, and intestine. This finding seemed to be particularly relevant, considering that the data were obtained from two independent laboratories ${ }^{25,26}$ and microarrays platforms. Regarding differentially expressed proteins, very few candidates (less than five) were identified as commonly modulated in at least two different tissues (heart, aortic, hepatic, and adipose tissues) and none was common to all tissues. Proteomic data were generated from three different laboratories. ${ }^{22,42,43}$ In silico analysis allowed us to identify 18 genes and a reduced number of proteins, which were subsequently tested for validation in rodent liver samples. Knowing that the liver is crucial for lipid metabolism and that samples were available from all studies, our hypothesis was that consistent modulation of specific targets of HT could be found in this tissue in animals supplemented with HT.

The transcriptomics studies included in this work were performed in five different tissues: breast, adipose tissue, intestine, liver, and brain. Among the four commonly differentially regulated genes selected for validation, Efr3a was down-regulated in all tissues, except for the intestine. Plscr1 was downregulated in all tissues, whereas Kctd2 was only downregulated in the intestine and liver and Slc37a4 was upregulated in the brain and adipose tissue. Validation of these four genes in the liver samples from the selected studies (Studies 1-4) confirmed the upregulation of Slc37a4 in Studies 2 and 3. The other genes did not change significantly, suggesting a large inter-study variability. None of the 14 additionally selected genes for validation (Fig. 1) changed in all four studies. While some genes only changed in one study (Anks6, Plscr2, Lipe, Snx16, Ppp1cb, Top1 and Tip2), others changed in two studies (Sort1, Slc37a4, Rora and Fgf21). According to our results, Study 4 showed the most reduced changes in gene expression, but we do not know whether this is related to the fact that this study was performed in rats rather than in mice. Moreover, in contrast with the other three mice studies receiving HT, in this study rats received secoiridoids. ${ }^{22}$ Secoiridoids are the major precursors of HT, after their in vivo digestion. ${ }^{2}$ Regarding the function of these genes, Sort1 influences plasma lipid concentration. ${ }^{44}$ Slc37a4 is a glucose-6-phosphate translocase (G6PT), which transports G6P from the cytoplasm into the endoplasmic reticulum (ER) lumen, and is involved in glucose metabolism. ${ }^{45}$ Rora is a nuclear receptor involved in multiple biological processes, including lipid metabolism. ${ }^{46} \mathrm{Fg} f 21$ is a metabolic gene that influences plasma glucose and triglyceride levels, ${ }^{47}$ and is a critical regulator of liver lipid homeostasis. ${ }^{48}$ Moreover, FGF21 is induced directly by PPARalpha in the liver in response to fasting ${ }^{49}$ and its induction is required for the normal activation of hepatic lipid oxidation and triglyceride clearance. $^{48}$ As such, induced expression of Fgf21 by HT feeding could be beneficial against metabolic diseases. Because Fgf21 and Rora are important contributors to metabolic diseases, we further validated their response to HT supplementation in a different cohort. $^{20}$ Interestingly, C57Bl6J mice receiving a single ingestion of HT dramatically increased their hepatic expression of $F g f 21$ at 1, 2, and 4 h post-ingestion. This effect was also observed for Rora, but to a lower degree $4 \mathrm{~h}$ post-ingestion (Fig. 6). In this sense, our bioinformatic approach and further validation uncovered novel possible molecular targets of the beneficial effects of HT consumption. A previous study performed in a different mouse model showed that the repression of $F g f 21$ caused by a high fat diet was reverted by HT supplementation. ${ }^{50}$ These and our current data suggest $F g f 21$ to be a bona fide candidate target of HT. Whether this effect occurs in humans is unknown and deserves further investigation.

In the study by Tomé-Carneiro et al., 2017 where samples deriving from Study 1 were used, a decrease in the expression of FASN and PRDX1 was recorded, by both high throughput proteomics and $\mathrm{WB}$, in mice supplemented with HT for eight weeks (Tomé-Carneiro et al., 2017). ${ }^{42}$ However, WB revealed only non-significant changes for these proteins in the liver samples tested for validation. WB analysis also revealed nonsignificant changes for CAR3 in the studies used for validation, despite it being reported as upregulated by high throughput proteomics in Study 1.

According to high throughput proteomics, VIM was significantly changed in the adipose tissue samples from Study 1 and the aortic tissue from Study 4 . Here, however, WB analysis
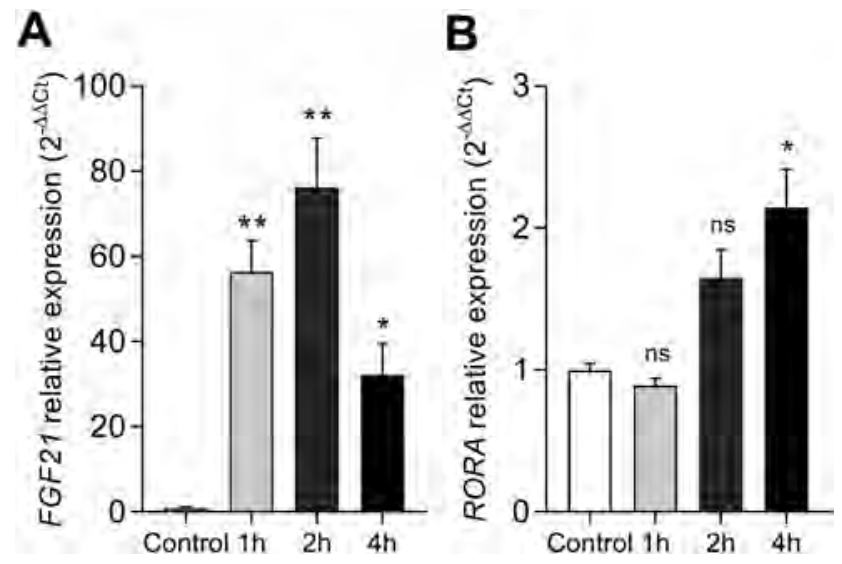

Fig. 6 Hydroxytyrosol target liver mRNA expression of Fgf21 and Rora. Effects of hydroxytyrosol on FGF21 (A) and RORA and (B) relative gene expression in liver samples of C57BI6J mice at different time points $(1,2$ and 4 hours). Data shown as mean \pm SEM. * $p<0.05$ compared to the control group; ${ }^{* *} p<0.0001$ compared to the control group. ( $n=9$ per group). 
showed non-significant changes for these proteins in the liver samples used for validation. The statistically significant changes observed for ACTN4 proteins in liver (Study 1), aortic, and heart tissues (Study 4), by WB, were not confirmed in the liver samples used for validation. Likewise, the statistically significant changes observed for HSPD1 in adipose (Study 1), hepatic (Study 3) and aortic (Study 4) tissues were not confirmed, by WB, in the liver samples used for validation. Overall, none of the proteins selected for validation showed consistent differential expression across the liver samples tested here.

It is important to note that the transcriptional data analysis was performed independently of the protein data levels and that correlations between transcripts and proteins were not intended during the validation process. Also, it is relevant to mention that the list of common genes or proteins to be validated were common to at least two different studies, regardless of whether the tissues subjected to high throughput analysis matched or not. Indeed, in most cases, tissues from where transcript (intestine, liver, adipose, brain or breast tumor) and protein (liver, heart, aortic, or adipose) levels were selected did not match. Thus, the lack of consistent validation of potential targets of HT in response to dietary supplementation should be seen with caution.

Other aspects of the complex regulatory variation from RNA to protein may account for the lack of common tissue features in response to HT supplementation. For instance, studies in model organisms and humans have shown that variations in mRNA and protein expression levels are often uncorrelated. ${ }^{51,52}$ Few transcripts are exclusive to a particular tissue and varies more across tissues than individuals, ${ }^{53}$ while genetic variation can also influence the heterogenicity of the protein expression in a diverse set of human tissues. ${ }^{52,54}$ Moreover, differences may also arise from alterations in posttranslational regulation. Regulation by ncRNAs, particularly miRNAs, is among the plethora of posttranslational controlling pathways.

Although in the past few years increasing evidence has suggested that food bioactive compounds can modulate the expression of miRNAs in vitro, ${ }^{55}$ in animal models, ${ }^{56}$ and in humans, ${ }^{57}$ very few studies have specifically focused on the action of HT. For example, specific miRNAs, miR-9 ${ }^{58}$ and miR-146a, ${ }^{59}$ were evaluated in vitro, whereas only one study evaluated the whole miRNome in the mouse small intestine. ${ }^{20}$ Among the liver modulated miRNAs in response to HT supplementation, miR-802-5p has been previously described as being obesity-inducing and as being involved in glucose metabolism impairment and in angiotensin signaling regulation. ${ }^{60,61}$ As for miR-423-3p, its levels have been positively associated with cell growth in liver, colon or other types of cancers. ${ }^{62,63}$ Induction of miR-30a-5p has been previously described to ameliorate liver fibrosis ${ }^{64}$ or to suppress breast tumor growth and metastasis. ${ }^{64,65}$ miR-146b has been shown to attenuate non-alcoholic steatohepatitis, ${ }^{66}$ although its down-regulation has been shown to promote cancer growth and metastasis. ${ }^{67}$
Sustained intake of HT at dietary doses by mice resulted in altered miRNA expression in the intestine (assessed in Study 1) and the liver (assessed here). Of note, HT supplementation resulted only in a consistent $(p<0.05)$ regulation of miR-802$5 p$ in both tissues (ESI Fig. S1†). The reduced number of common modulated miRNAs found could be explained by different aspects of miRNA biogenesis, function, and technical analysis. For example, some miRNAs are tissue-specific. ${ }^{68}$ Also, though the processing pattern of miRNAs in tissues and cell lines may differ, it has been reported that, especially in cell lines, several transcribed miRNAs are not processed to mature miRNA. ${ }^{69}$ While miRNAs might play a role in the biological action of HT, the very reduced number of HT studies evaluating miRNAs precludes any conclusion regarding their regulatory potential at this point. However, the consistent induction of miR-802-5p in two different tissues, in response to dietary HT supplementation, seems to support a miRNA modulating action of small natural molecules, which could be exploited as a potential therapeutic alternative or adjuvant to the current pharmacological arsenal targeting endogenous miRNAs.

\section{Conclusions}

High throughput transcriptomics and proteomics are powerful tools that greatly contribute to the knowledge of how nutrition affects the expression of a wide number of genes and proteins. Although in vivo studies where these techniques are employed to investigate the molecular effects of hydroxytyrosol are increasing in number, they are still scarce. Therefore, we believe that there is a growing need to integrate the accumulating data in order to identify the consistent targets of this bioactive compound. Most of the genes and proteins identified and tested here as potential HT targets showed inconsistent modulation by HT. These results are, at least in part, due to the limited number of in vivo studies available, with heterogeneous HT doses and supplementation times, where different tissues were used for transcriptomic/proteomic analysis. However, our transcriptomic analysis uncovered two novel potential HT target candidates, i.e. Fgf21 and Rora. While we certainly do not want to depreciate the important role of omics and their related database, we feel that more attention should be paid to the current pitfalls of this approach to nutritional research. Over-emphasis should be avoided and more HT-supplementation studies employing high throughput transcriptomics and proteomics tools are needed for potential HT targets to be identified and validated.

\section{Author contributions}

MCLH, RMH and MCC contributed equally to this work. AD, MCLH and FV contributed to the conception or design of the work. RMH, MCC, JT-C, MB R-R and LdP contributed to data collection. LR, MJM, JO and MN contributed with samples from different studies. AD, FV, JAM and MPP obtained finan- 
cial support. MCC, JT-C, MCLH, RMH, AD and FV drafted the article. JAM, JO, JCE-G and MPP revised the manuscript for important intellectual contribution. All authors reviewed and approved the manuscript.

\section{Abbreviations}
HT Hydroxytyrosol
GO Gene ontology
GI Gene Interaction

\section{Conflicts of interest}

The authors declare no conflicts of interest related to this work.

\section{Acknowledgements}

This research was funded by grants from the Spanish "Agencia Estatal de Investigación" and the European FEDER Funds to $\mathrm{AD}$ and RMH (AGL2016-78922-R); the Ministerio de Economía y Competitividad-Fondo Europeo de Desarrollo Regional (SAF2016-75441-R) and the Fondo Social Europeo-Gobierno de Aragón (B16_17R) to MAN and JO; the Fundación Ramón Areces (CIVP18A3888) to AD, JG-Z, JTC, MCC, FV and RMH; the CIBER de Fisiopatología de la Obesidad y Nutrición (CIBERObn) to JAM, MPP, JO and MAN; and POR FESR 3S4H to FV. CIBERObn. is an initiative of the ISCIII, Spain. MCLH, LdP and MB R-R were recipients of contracts from the Consejería de Educación, Juventud y Deporte de la Comunidad de Madrid, Fondo Social Europeo, and the Iniciativa de Empleo Juvenil YEI (PEJD-2016/BIO-2781, PEJD-2017-PRE/ BIO-5100, and PEJD-2018-POST/BIO 8933), respectively.

\section{References}

1 M. C. L. de las Hazas, L. Rubio, A. Macia and M. J. Motilva, Hydroxytyrosol: Emerging Trends in Potential Therapeutic Applications, Curr. Pharm. Des., 2018, 24, 2157-2179.

2 M.-C. López de las Hazas, C. Piñol, A. Macià, M.-P. Romero, et al., Differential absorption and metabolism of hydroxytyrosol and its precursors oleuropein and secoiridoids, J. Funct. Foods, 2016, 22, 52-63.

3 L. Rubió, R.-M. Valls, A. Macià, A. Pedret, et al., Impact of olive oil phenolic concentration on human plasmatic phenolic metabolites, Food Chem., 2012, 135, 2922-2929.

4 M. de Bock, E. B. Thorstensen, J. G. B. Derraik, H. V. Henderson, et al., Human absorption and metabolism of oleuropein and hydroxytyrosol ingested as olive (Olea europaea L.) leaf extract, Mol. Nutr. Food Res., 2013, 57, 2079-2085.

5 O. Khymenets, M. C. Crespo, O. Dangles, N. Rakotomanomana, et al., Human hydroxytyrosol's absorption and excretion from a nutraceutical, J. Funct. Foods, 2016, 23, 278-282.

6 F. Visioli, A. Davalos, M. López de las Hazas, M. C. Crespo, et al., An overview of the pharmacology of olive oil and its active ingredients, Br. J. Pharmacol., 2019, DOI: 10.1111/ bph.14782.

7 M. Crespo, J. Tomé-Carneiro, A. Dávalos and F. Visioli, Pharma-Nutritional Properties of Olive Oil Phenols. Transfer of New Findings to Human Nutrition, Foods, 2018, 7, 90.

8 R. Valenzuela, F. Echeverria, M. Ortiz, M.Á Rincón-Cervera, et al., Hydroxytyrosol prevents reduction in liver activity of $\Delta-5$ and $\Delta-6$ desaturases, oxidative stress, and depletion in long chain polyunsaturated fatty acid content in different tissues of high-fat diet fed mice, Lipids Health Dis., 2017, 16, 64 .

9 S. Tejada, S. Pinya, M. Mar Bibiloni, J. A. del Tur, et al., Cardioprotective Effects of the Polyphenol Hydroxytyrosol from Olive Oil, Curr. Drug Targets, 2017, 18, 1477-1486.

10 N. Richard, S. Arnold, U. Hoeller, C. Kilpert, et al., Hydroxytyrosol is the major anti-inflammatory compound in aqueous olive extracts and impairs cytokine and chemokine production in macrophages, Planta Med., 2011, 77, 1890-1897.

11 A. Pedret, S. Fernández-Castillejo, R.-M. Valls, Ú. Catalán, et al., Cardiovascular Benefits of Phenol-Enriched Virgin Olive Oils: New Insights from the Virgin Olive Oil and Hdl Functionality (Vohf) Study, Mol. Nutr. Food Res., 2018, 1800456.

12 R. Martín-Hernández, G. Reglero and A. Dávalos, Data mining of nutrigenomics experiments: Identification of a cancer protective gene signature, J. Funct. Foods, 2018, 42, 380-386.

13 P. Suravajhala, L. J. A. Kogelman and H. N. Kadarmideen, Multi-omic data integration and analysis using systems genomics approaches: methods and applications in animal production, health and welfare, Genet., Sel., Evol., 2016, 48, 38.

14 D. Braconi, G. Bernardini, L. Millucci and A. Santucci, Foodomics for human health: current status and perspectives, Expert Rev. Proteomics, 2018, 15, 153-164.

15 C. Pavlidis, Z. Lanara, A. Balasopoulou, J.-C. Nebel, et al., Meta-Analysis of Genes in Commercially Available Nutrigenomic Tests Denotes Lack of Association with Dietary Intake and Nutrient-Related Pathologies, OMICS: J. Integr. Biol., 2015, 19, 512-520.

16 P. A. Maranhão, G. M Bacelar-Silva, D. N. G. Ferreira, C. Calhau, et al., Nutrigenomic Information in the openEHR Data Set, Appl. Clin. Inform., 2018, 9, 221-231.

17 F. Vitali, R. Lombardo, D. Rivero, F. Mattivi, et al., ONS: an ontology for a standardized description of interventions and observational studies in nutrition, Genes Nutr., 2018, 13,12 .

18 S. Dhanasekaran, T. K. Bhattacharya, R. N. Chatterjee, C. Paswan, et al., Functional genomics in chicken (Gallus gallus) - status and implications in poultry, Worlds. Poult. Sci. J., 2014, 70, 45-56. 
19 R. Agarwal and V. Dhar, Editorial-Big Data, Data Science, and, Analytics: The Opportunity and Challenge for IS Research, Inf. Syst. Res., 2014, 25, 443-448.

20 J. Tomé-Carneiro, M. C. Crespo, E. Iglesias-Gutierrez, R. Martín, et al., Hydroxytyrosol supplementation modulates the expression of miRNAs in rodents and in humans, J. Nutr. Biochem., 2016, 34, 146-155.

21 S. Acín, M. A. Navarro, J. M. Arbonés-Mainar, N. Guillén, et al., Hydroxytyrosol administration enhances atherosclerotic lesion development in apo $\mathrm{E}$ deficient mice, J. Biochem., 2006, 140, 383-391.

22 Ú. Catalán, L. Rubió, M.-C. López de las Hazas, P. Herrero, et al., Hydroxytyrosol and its complex forms (secoiridoids) modulate aorta and heart proteome in healthy rats: Potential cardio-protective effects, Mol. Nutr. Food Res., 2016, 60, 2114-2129.

23 C. Sticht, C. De La Torre, A. Parveen and N. Gretz, miRWalk: An online resource for prediction of microRNA binding sites, PLoS One, 2018, 13, e0206239.

24 H. Mi, X. Huang, A. Muruganujan, H. Tang, et al., PANTHER version 11: expanded annotation data from Gene Ontology and Reactome pathways, and data analysis tool enhancements, Nucleic Acids Res., 2017, 45, D183-D189.

25 S. Granados-Principal, J. L. Quiles, C. Ramirez-Tortosa, P. Camacho-Corencia, et al., Hydroxytyrosol inhibits growth and cell proliferation and promotes high expression of sfrp4 in rat mammary tumours, Mol. Nutr. Food Res., 2011, 55(Suppl 1), S117-S126.

26 E. Giordano, A. Dávalos and F. Visioli, Chronic hydroxytyrosol feeding modulates glutathione-mediated oxidoreduction pathways in adipose tissue: A nutrigenomic study, Nutr., Metab. Cardiovasc. Dis., 2014, 24, 1144-1150.

27 E. C. Lai, Micro RNAs are complementary to 3' UTR sequence motifs that mediate negative post-transcriptional regulation, Nat. Genet., 2002, 30, 363-364.

28 D. Baek, J. Villén, C. Shin, F. D. Camargo, et al., The impact of microRNAs on protein output, Nature, 2008, 455, 64-71.

29 J. M. Ordovas and D. Corella, Nutritional genomics., Annu. Rev. Genomics Hum. Genet., 2004, 5, 71-118.

30 A. P. Simopoulos, Nutrigenetics/Nutrigenomics, Annu. Rev. Public Health, 2010, 31, 53-68.

31 H. Nakayama, Y. Shimada, L. Zang, M. Terasawa, et al., Novel Anti-Obesity Properties of Palmaria mollis in Zebrafish and Mouse Models, Nutrients, 2018, 10, 1401.

32 A. Alkhatib, C. Tsang and J. Tuomilehto, Olive Oil Nutraceuticals in the Prevention and Management of Diabetes: From Molecules to Lifestyle, Int. J. Mol. Sci., 2018, 19, 2024.

$33 \mathrm{~J}$. Logan and M. W. Bourassa, The rationale for a role for diet and nutrition in the prevention and treatment of cancer, Eur. J. Cancer Prev., 2018, 27, 406-410.

34 M.-H. Pan, J.-C. Wu, C.-T. Ho and C.-S. Lai, Antiobesity molecular mechanisms of action: Resveratrol and pterostilbene, BioFactors, 2018, 44, 50-60.

35 L. Väremo, C. Scheele, C. Broholm, A. Mardinoglu, et al., Proteome- and transcriptome-driven reconstruction of the human myocyte metabolic network and its use for identification of markers for diabetes, Cell Rep., 2015, 11, 921-933.

36 V. do Nascimento de Oliveira, A. B. M. Lima-Neto, M. F. van Tilburg, A. C. de Oliveira Monteiro-Moreira, et al., Proteomic analysis to identify candidate biomarkers associated with type 1 diabetes, Diabetes, Metab. Syndr. Obes., 2018, 11, 289-301.

37 X. Yan, Y. Wu, F. Zhong, Q. Jiang, et al., iTRAQ and PRMbased quantitative proteomics in T2DM-susceptible and -tolerant models of Bama mini-pig, Gene, 2018, 675, 119127.

38 M. Murri, M. Insenser, M. R. Bernal-Lopez, P. PerezMartinez, et al., Proteomic analysis of visceral adipose tissue in pre-obese patients with type 2 diabetes, Mol. Cell. Endocrinol., 2013, 376, 99-106.

39 B. de Roos and D. F. Romagnolo, Proteomic Approaches to Predict Bioavailability of Fatty Acids and Their Influence on Cancer and Chronic Disease Prevention, J. Nutr., 2012, 142, 1370S-1376S.

40 Y. E. Kim, H. J. Jeon, D. Kim, S. Y. Lee, et al., Quantitative Proteomic Analysis of 2D and 3D Cultured Colorectal Cancer Cells: Profiling of Tankyrase Inhibitor XAV939Induced Proteome, Sci. Rep., 2018, 8, 13255.

41 A. Aliper, S. Plis, A. Artemov, A. Ulloa, et al., Deep Learning Applications for Predicting Pharmacological Properties of Drugs and Drug Repurposing Using Transcriptomic Data, Mol. Pharm., 2016, 13, 2524-2530.

42 J. Tomé-Carneiro, M. C. Crespo, E. García-Calvo, J. L. Luque-García, et al., Proteomic evaluation of mouse adipose tissue and liver following hydroxytyrosol supplementation, Food Chem. Toxicol., 2017, 107, 329-338.

43 G. Rodríguez-Gutiérrez, G. G. Duthie, S. Wood, P. Morrice, et al., Alperujo extract, hydroxytyrosol, and 3,4-dihydroxyphenylglycol are bioavailable and have antioxidant properties in vitamin E-deficient rats-a proteomics and network analysis approach, Mol. Nutr. Food Res., 2012, 56, 1137-1147.

44 C. J. Willer, S. Sanna, A. U. Jackson, A. Scuteri, et al., Newly identified loci that influence lipid concentrations and risk of coronary artery disease, Nat. Genet., 2008, 40, 161-169.

45 A. R. Cappello, R. Curcio, R. Lappano, M. Maggiolini, et al., The Physiopathological Role of the Exchangers Belonging to the SLC37 Family, Front. Chem., 2018, 6, 122.

46 K. Kim, K. Boo, Y. S. Yu, S. K. Oh, et al., ROR $\alpha$ controls hepatic lipid homeostasis via negative regulation of PPAR $\gamma$ transcriptional network, Nat. Commun., 2017, 8, 162.

47 A. Kharitonenkov, T. L. Shiyanova, A. Koester, A. M. Ford, et al., FGF-21 as a novel metabolic regulator, J. Clin. Invest., 2005, 115, 1627-1635.

48 M. K. Badman, P. Pissios, A. R. Kennedy, G. Koukos, et al., Hepatic Fibroblast Growth Factor 21 Is Regulated by PPAR $\alpha$ and Is a Key Mediator of Hepatic Lipid Metabolism in Ketotic States, Cell Metab., 2007, 5, 426-437.

49 T. Inagaki, P. Dutchak, G. Zhao, X. Ding, et al., Endocrine Regulation of the Fasting Response by PPAR $\alpha$-Mediated Induction of Fibroblast Growth Factor 21, Cell Metab., 2007, 5, 415-425. 
50 C. Pirozzi, A. Lama, R. Simeoli, O. Paciello, et al., Hydroxytyrosol prevents metabolic impairment reducing hepatic inflammation and restoring duodenal integrity in a rat model of NAFLD, J. Nutr. Biochem., 2016, 30, 108-115.

51 E. J. Foss, D. Radulovic, S. A. Shaffer, D. M. Ruderfer, et al., Genetic basis of proteome variation in yeast, Nat. Genet., 2007, 39, 1369-1375.

52 A. Battle, Z. Khan, S. H. Wang, A. Mitrano, et al., Impact of regulatory variation from RNA to protein, Science, 2015, 347, 664-667.

53 M. Mele, P. G. Ferreira, F. Reverter, D. S. DeLuca, et al., The human transcriptome across tissues and individuals, Science, 2015, 348, 660-665.

54 GTEx Consortium, Gte., Human genomics. The GenotypeTissue Expression (GTEx) pilot analysis: multitissue gene regulation in humans, Science, 2015, 348, 648-660.

55 L. Baselga-Escudero, C. Blade, A. Ribas-Latre, E. Casanova, et al., Resveratrol and EGCG bind directly and distinctively to miR-33a and miR-122 and modulate divergently their levels in hepatic cells, Nucleic Acids Res., 2014, 42, 882-892.

56 D. Milenkovic, C. Deval, E. Gouranton, J.-F. Landrier, et al., Modulation of miRNA Expression by Dietary Polyphenols in apoE Deficient Mice: A New Mechanism of the Action of Polyphenols, PLoS One, 2012, 7, e29837.

57 M. A. Nuñez-Sánchez, A. Dávalos, A. González-Sarrías, P. Casas-Agustench, et al., MicroRNAs expression in normal and malignant colon tissues as biomarkers of colorectal cancer and in response to pomegranate extracts consumption: Critical issues to discern between modulatory effects and potential artefacts, Mol. Nutr. Food Res., 2015, 59, 1973-1986.

58 S. D’Adamo, S. Cetrullo, S. Guidotti, R. M. Borzì, et al., Hydroxytyrosol modulates the levels of microRNA-9 and its target sirtuin-1 thereby counteracting oxidative stressinduced chondrocyte death, Osteoarthritis Cartilage, 2017, 25, 600-610.

59 E. Bigagli, L. Cinci, S. Paccosi, A. Parenti, et al., Nutritionally relevant concentrations of resveratrol and hydroxytyrosol mitigate oxidative burst of human granulocytes and monocytes and the production of pro-inflammatory mediators in LPS-stimulated RAW 264.7 macrophages, Int. Immunopharmacol., 2017, 43, 147-155.

60 S. E. Sansom, G. J. Nuovo, M. M. Martin, S. R. Kotha, et al., miR-802 regulates human angiotensin II type 1 receptor expression in intestinal epithelial C2BBe1 cells, Am. J. Physiol.: Gastrointest. Liver Physiol., 2010, 299, G632-G642.

61 J.-W. Kornfeld, C. Baitzel, A. C. Könner, H. T. Nicholls, et al., Obesity-induced overexpression of miR-802 impairs glucose metabolism through silencing of Hnf1b, Nature, 2013, 494, 111-115.

62 H.-T. Li, H. Zhang, Y. Chen, X.-F. Liu, et al., MiR-423-3p enhances cell growth through inhibition of p21Cip1/Waf1 in colorectal cancer, Cell. Physiol. Biochem., 2015, 37, 1044-1054.

63 J. Lin, S. Huang, S. Wu, J. Ding, et al., MicroRNA-423 promotes cell growth and regulates $\mathrm{G} 1$ /S transition by targeting p21Cip1/Waf1 in hepatocellular carcinoma, Carcinogenesis, 2011, 32, 1641-1647.

64 J. Chen, Y. Yu, S. Li, Y. Liu, et al., MicroRNA-30a ameliorates hepatic fibrosis by inhibiting Beclin1-mediated autophagy, J. Cell. Mol. Med., 2017, 21, 3679-3692.

65 L. Li, L. Kang, W. Zhao, Y. Feng, et al., miR-30a-5p suppresses breast tumor growth and metastasis through inhibition of LDHA-mediated Warburg effect, Cancer Lett., 2017, 400, 89-98.

66 W. Jiang, J. Liu, Y. Dai, N. Zhou, et al., MiR-146b attenuates high-fat diet-induced non-alcoholic steatohepatitis in mice, J. Gastroenterol. Hepatol., 2015, 30, 933-943.

67 C. Li, R. Miao, S. Liu, Y. Wan, et al., Down-regulation of miR-146b-5p by long noncoding RNA MALAT1 in hepatocellular carcinoma promotes cancer growth and metastasis, Oncotarget, 2017, 8, 28683-28695.

68 M. Lagos-Quintana, R. Rauhut, A. Yalcin, J. Meyer, et al., Identification of tissue-specific microRNAs from mouse, Curr. Biol., 2002, 12, 735-739.

69 E. J. Lee, M. Baek, Y. Gusev, D. J. Brackett, et al., Systematic evaluation of microRNA processing patterns in tissues, cell lines, and tumors, $R N A, 2008,14,35-42$.

70 A. Voigt, J. Ribot, A. G. Sabater, A. Palou, et al., Identification of Mest/Peg1 gene expression as a predictive biomarker of adipose tissue expansion sensitive to dietary anti-obesity interventions, Genes Nutr., 2015, 10, 27.

71 A. Zheng, H. Li, J. Xu, K. Cao, et al., Hydroxytyrosol improves mitochondrial function and reduces oxidative stress in the brain of $\mathrm{db} / \mathrm{db}$ mice: role of AMP-activated protein kinase activation, Br. J. Nutr., 2015, 113, 1667-1676.

72 A. Zheng, H. Li, K. Cao, J. Xu, et al., Maternal hydroxytyrosol administration improves neurogenesis and cognitive function in prenatally stressed offspring, J. Nutr. Biochem., 2015, 26, 190-199. 